INFN-14-22/LNF

December 25th, 2014

\title{
PERFORMANCE OF THE GAS GAIN MONITORING SYSTEM OF THE CMS RPC MUON DETECTOR
}

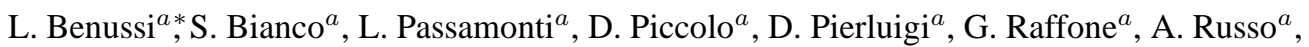

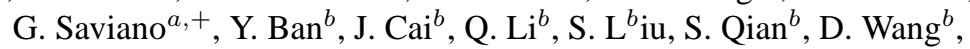

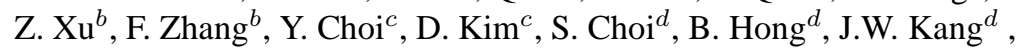
M. Kang ${ }^{d}$, J.H. Kwon ${ }^{d}$, K.S. Lee, ${ }^{d}$, S.K. Park ${ }^{d}$, L. Pant ${ }^{e}$, V.B.J. $\operatorname{Singh}^{f}$, A.M.R. Kumar ${ }^{f}$,

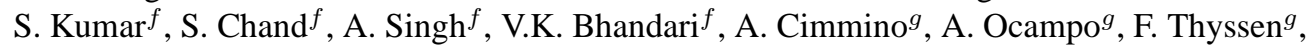
M. Tytgat ${ }^{g}$, W. Van Doninck ${ }^{h}$, A. Ahmad ${ }^{i}$, S. Muhamma ${ }^{i}$, M. Shoaib ${ }^{i}$, H. Hoorani ${ }^{i}$, I. Awan, ${ }^{i}$

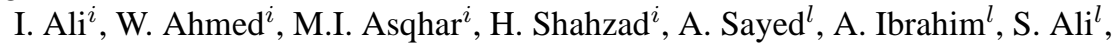
R. Ali ${ }^{l}$, A. Radi ${ }^{l}$, T. Elkafrawi ${ }^{l}$, A. Sharma ${ }^{m}$, S. Colafranceschi ${ }^{m}$, M. Abbrescia ${ }^{n}$,

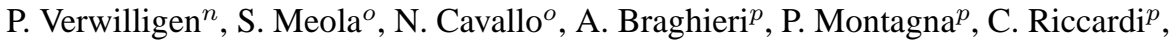

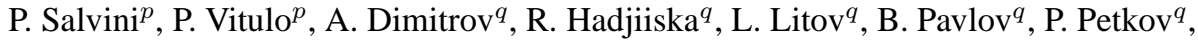

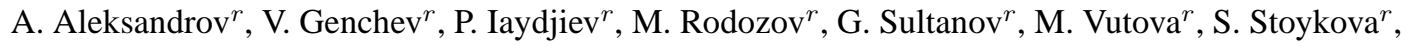
H.S. Ibargüen ${ }^{s}$, M.I. Pedraza Morales ${ }^{s}$, S. Carpinteyro Bernardino ${ }^{s}$, I. Bagaturia ${ }^{t}$, Z. Tsamalaidze ${ }^{t}$.

\begin{abstract}
The RPC muon detector of the CMS experiment at the LHC (CERN, Geneva, Switzerland) is equipped with a Gas Gain Monitoring (GGM) system. A report on the stability of the system during the 2011-2012 data taking run is given, as well as the observation of an effect which suggests a novel method for the monitoring of gas mixture composition.
\end{abstract}

Corresponding author. 
Submitted to JINST. Keywords: CMS; RPC; muon detector; gas detector

\author{
${ }^{a}$ Laboratori Nazionali di Frascati dell'INFN, Frascati, Italy \\ ${ }^{b}$ Peking University, Peking, China \\ ${ }^{c}$ SKK University, Seoul, S. Korea \\ ${ }^{d}$ Korea University, Seoul, S. Korea \\ ${ }^{e}$ BARC, Mumbai, India \\ ${ }^{f}$ Chandigarh University, India \\ ${ }^{g}$ Ghent University, Ghent, Belgium \\ ${ }^{h}$ VUB, Bruxelles, Belgium \\ ${ }^{i} \mathrm{NCP}$, Islamabad, Pakistan \\ ${ }^{l}$ ENHEP, Cairo, Egypt \\ ${ }^{m}$ CERN, Geneve, Switzerland \\ ${ }^{n}$ Università di Bari and INFN Bari, Bari, Italy \\ ${ }^{\circ}$ Università di Napoli and INFN Napoli, Napoli, Italy \\ ${ }^{p}$ Università di Pavia and INFN Pavia, Pavia, Italy \\ ${ }^{q}$ University of Sofia, Sofia, Bulgaria \\ ${ }^{r}$ INRNE, Sofia, Bulgaria \\ ${ }^{s}$ Puebla University, Puebla, Mexico \\ ${ }^{t}$ Tbilisi University and IHEPI, Tbilisi, Georgia \\ ${ }^{+}$also at Università degli Studi di Roma La Sapienza, Rome, Italy \\ *E-mail: luigi. benussi@lnf.infn.it
}




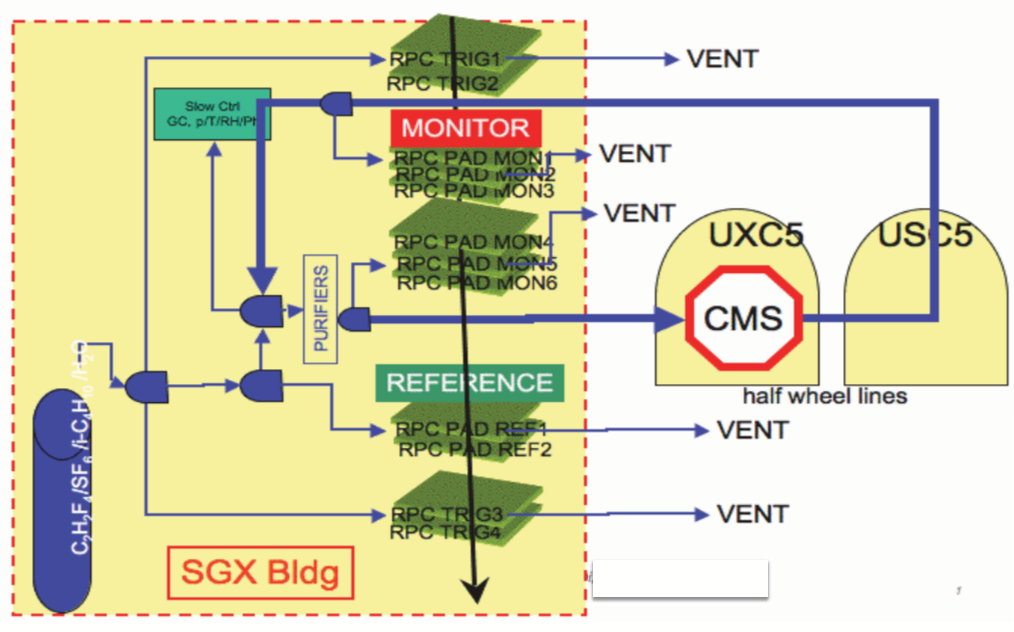

Figure 1: Schematic layout of the GGM and closed-loop gas recirculation system of the CMS RPC detector.

\section{Introduction}

The Resistive Plate Counter (RPC) muon detector of the Compact Muon Solenoid (CMS) experiment at the Large Hadron Collider (LHC) at CERN is equipped with a Gas Gain Monitoring (GGM) system. Detailed descriptions of the GGM can be found in [1,?, ?, ?]. The system has been in operation for the whole duration of the 2011-2012 CMS data taking period. A report of both its performance and the experience gained with it is given. An effect which may suggest a novel method for the monitoring of gas mixture composition is discussed.

\section{Experimental setup}

The GGM system is composed of 12 square single-gap RPC detectors arranged as a cosmic ray hodoscope (Fig 1). The GGM is located on the surface, in the SGX5 gas room of the CMS experimental area.

The system is designed to provide a fast and accurate determination of any shift in the working point of its chambers due to gas mixture changes. It compares three different gas mixtures from the CMS experiment: a newly supplied fresh gas mixture, and the gas mixture before and after the filters of the closed-loop recirculation system. The closed loop is fed with one volume $\left(14 \mathrm{~m}^{3}\right)$ gas exchange per hour [ [5].

The 12 single bakelite gaps have a double pad readout (4 trigger gaps, 8 signal gaps) which allows removal of coherent noise (i.e., the environmental baseline noise showing on both pads) by subtracting algebraically signals from both pads. The average event rate is $5 \mathrm{~Hz}$, corresponding to about 30 minutes for 10000 events. Anode charge distributions are collected for blocks of 30 minutes, and changes of the 
charge averages over time provide indications of changes in the working point of the GGM chambers. An automatic compensation of environmental effects on the chamber responses is achieved by means of two-gap ratios of anode charge distributions (Fig 2] [2].

\subsection{High-voltage scans}

The GGM system was designed to detect changes in the working point of the chambers via differences between a fresh gas mixture, and the mixtures before and after the purifiers of the gas recirculation system, using by also the charge ratio algorithm. Next to that, weekly RPC high voltage (HV) scans were performed which additionally provided a direct measurement of the GGM working point, allowing to spot changes in the gas mixture composition.

Fig. 3 shows typical HV scans of two GGM single-gap chambers, as a function of effective HV supply

$$
H V_{\text {eff }}=H V \frac{p_{0}}{p} \frac{T}{T_{0}}
$$

The $H V_{\text {eff }}$ value corresponding to the effective voltage where the chamber efficiency $\epsilon$ is at $50 \%$ of its maximum

$$
H V 50 \equiv H V_{e f f}\left(\epsilon=\epsilon_{\max } / 2\right)
$$

is the most sensitive parameter to any change of the working point.

The HV50 parameter is shown for a few GGM chambers in Fig. 4 as a function of time for the 2011-2012 data taking period.

Each HV50 value is sensitive to common environmental effects that are not corrected for by the HV feedback. A normalization method taking the ratio of the HV50 values for the gas mixtures in the closedloop system and the fresh gas mixture, provides a stable response over a period of several months (Fig [5). $\triangle H V 50$ is the difference in the HV50 parameter for the before- or after-type gaps normalized to the freshtype gaps. The distribution of $\Delta H V 50$ parameter over time is compatible with statistical errors and does not suggest any systematic effect that may indicate a possible problem on the gas mixture composition.

\subsection{Time transient}

An unexpected feature was observed thanks to a failure of one of the mass flow controllers (MFC) of the gas mixer (Fig. 6). The duration of each MFC failure was estimated in approximately 30 minutes. The pattern observed in all charge ratios is interpreted as the interference of the working point changes due to the gas mixture change, convoluted with the transit time of the gas mixture inside the closed-loop recirculation system. The fresh gas mixture reaches the GGM before both the affected gas mixtures from before and after the purifiers in the closed-loop system, thus causing the interference pattern shown in Fig. 7 


\section{Conclusions}

Preliminary results on the operational experience of the GGM system during the CMS 2011-2012 data taking period were given. Weekly HV scans provide direct measurements of the GGM working point. When corrected for common environmental effects, the system shows a stability in working point at the level of $20 \mathrm{~V}$ over nearly two years. A failure in one of the MFC provided hints to define a tool for the fast monitoring of the gas mixture composition. If confirmed, the gas mixture composition might be monitored by the time distribution pattern of the fresh-, before-, and after-purifier type gas mixture average charge ratios. A simulation analysis is in progress to verify such an interpetation.

\section{References}

[1] S. Colafranceschi, et al., "Performance of the Gas Gain Monitoring system of the CMS RPC muon detector and effective working point fine tuning," JINST 7 (2012) P12004 [PoS RPC 2012 (2012) 046] [arXiv:1209.3893 [hep-ex]].

[2] S. Colafranceschi, et al., "Operational experience of the gas gain monitoring system of the CMS RPC muon detectors," Nucl. Instrum. Meth. A 617 (2010) 146.

[3] L. Benussi, et al., "Sensitivity and environmental response of the CMS RPC gas gain monitoring system,” JINST 4 (2009) P08006 [arXiv:0812.1710 [physics.ins-det]].

[4] L. Benussi, et al., "The CMS RPC gas gain monitoring system: An Overview and preliminary results," Nucl. Instrum. Meth. A 602 (2009) 805 [arXiv:0812.1108 [physics.ins-det]].

[5] M. Capeans, R. Guida, F. Hahn, S. Haider and B. Mandelli, "RPC performances and gas quality in a closed loop gas systemfor the new purifiers configuration at LHC experiments," JINST 8, T08003 (2013). 

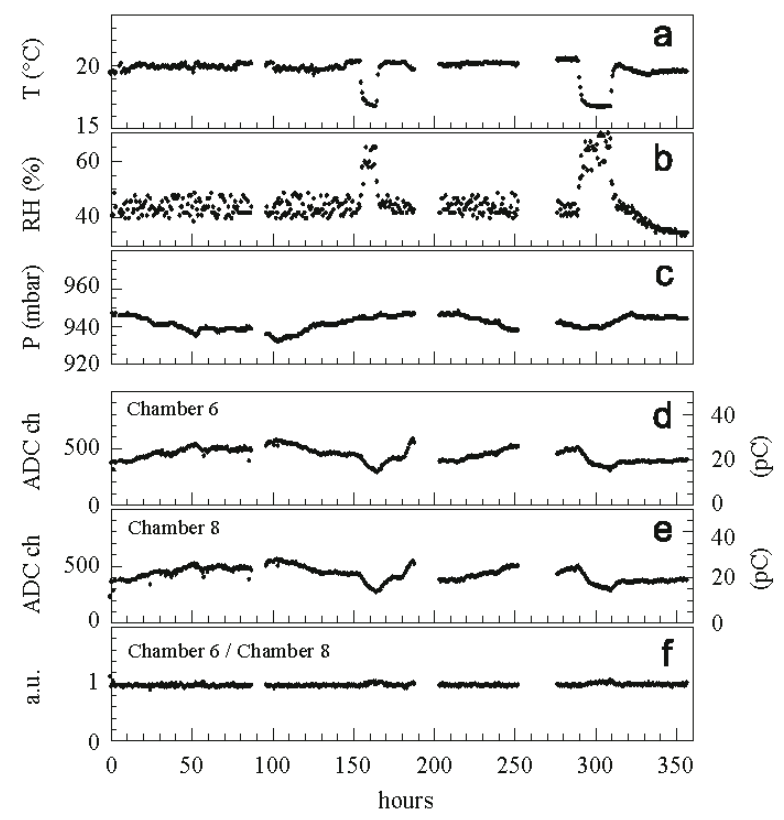

Figure 2: The principle of automatic compensation of environmental effects by charge ratios: environmental variables such as temperature (a), relative humidity (b), atmospheric pressure (c), gaps anodic average charges $(\mathrm{d}, \mathrm{e})$ and their ratio (f). 


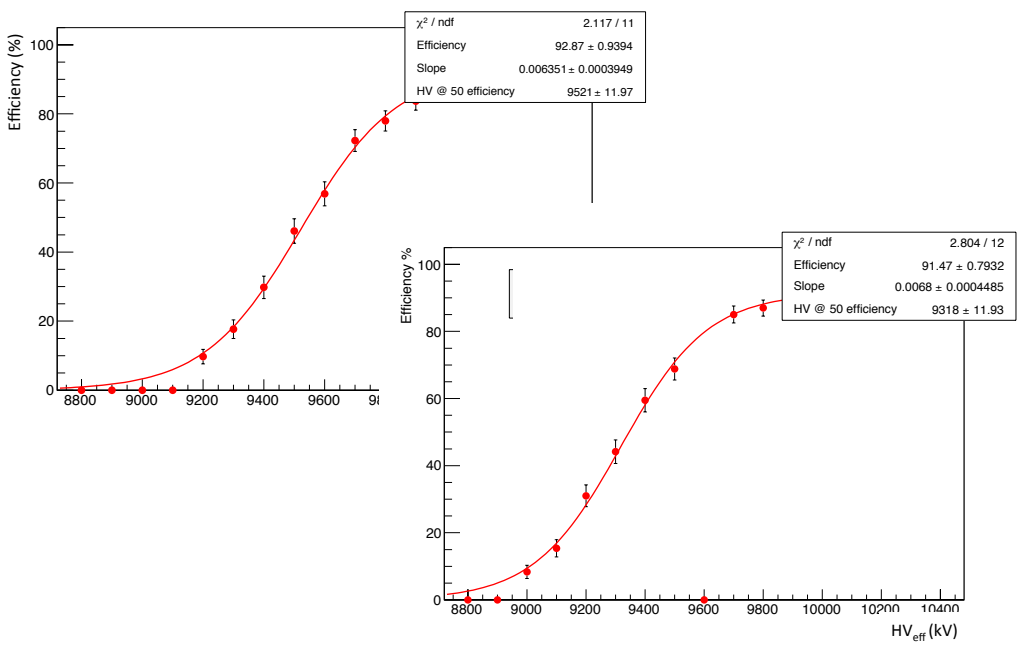

Figure 3: Typical HV scans for two GGM single-gap chambers.

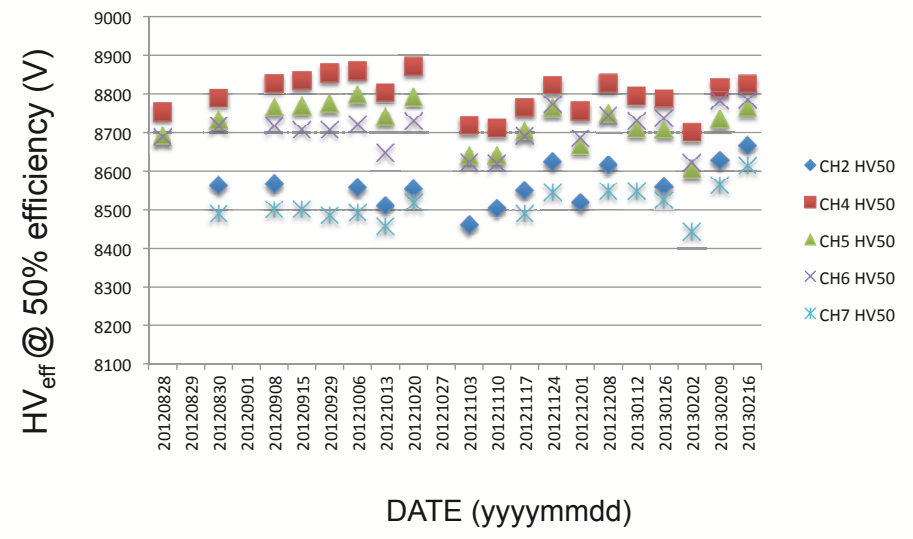

Figure 4: Dependence of HV50 (defined in text) as a function of time over the 2011-2012 data taking period. 


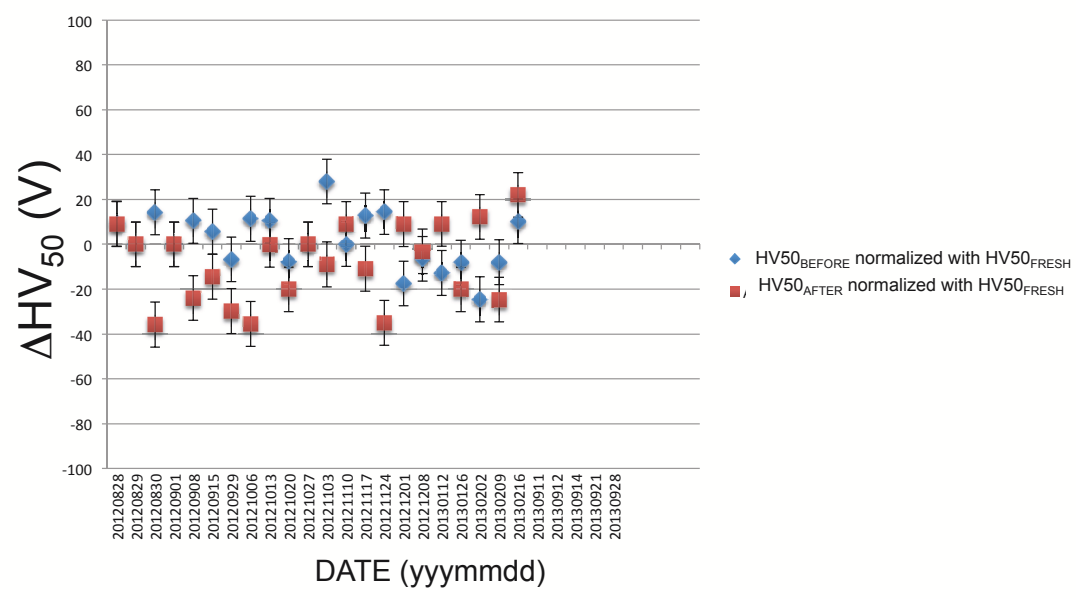

Figure 5: Dependence of normalized HV50.

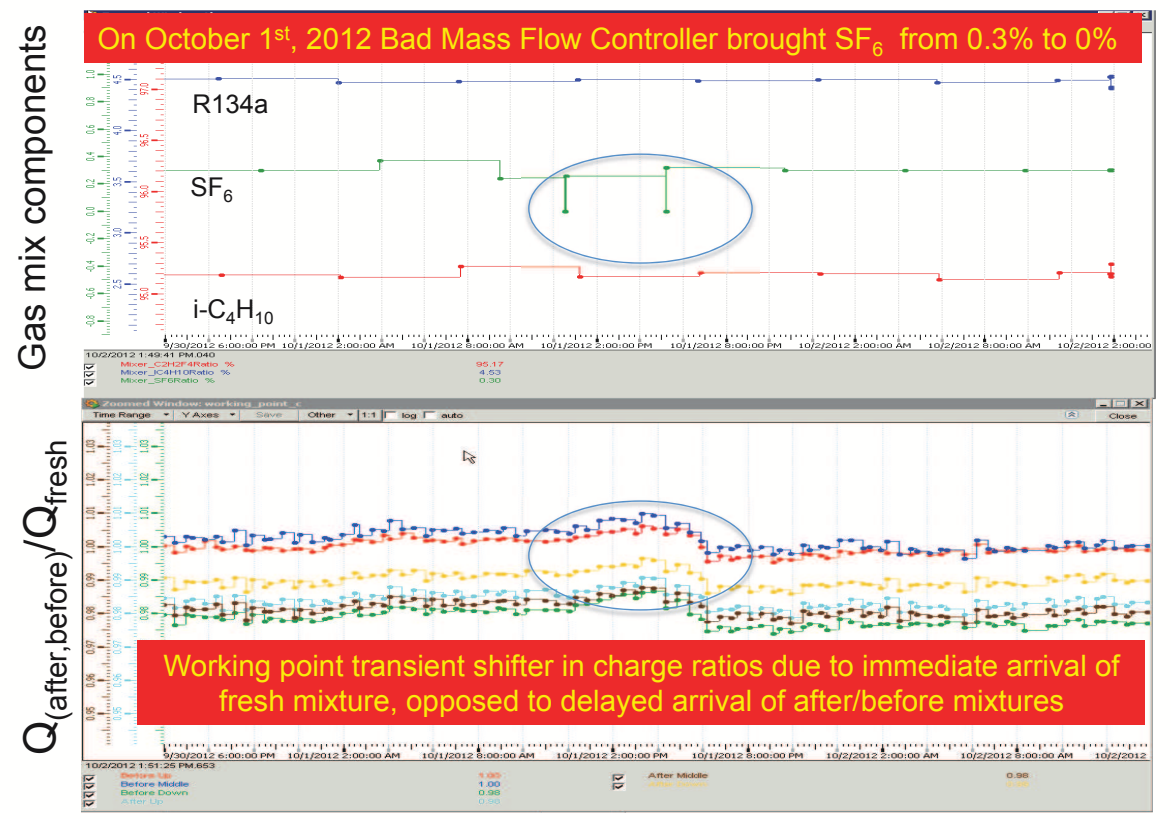

Figure 6: Failure of $S F_{6}$ mass flow controller (top); interference pattern observed in the online monitoring tool for all charge ratios of the GGM. 


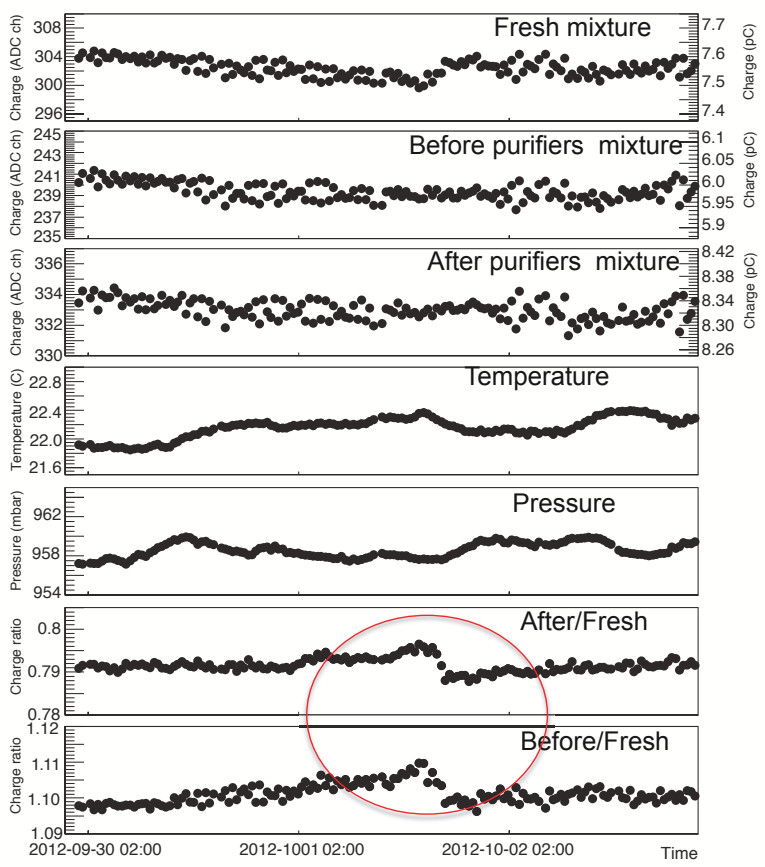

Figure 7: Analysis of the transient interference pattern and correlation with charges and environmental parameters. 